\title{
Automated Scenario Generation for Meeting Human-in-the-Loop Simulation Requirements
}

Gano Chatterji and Yun Zheng

Crown Consulting, Inc.

Moffett Field, CA 94035-1000 


\section{Air Traffic Management Testbed (ATMTB)}

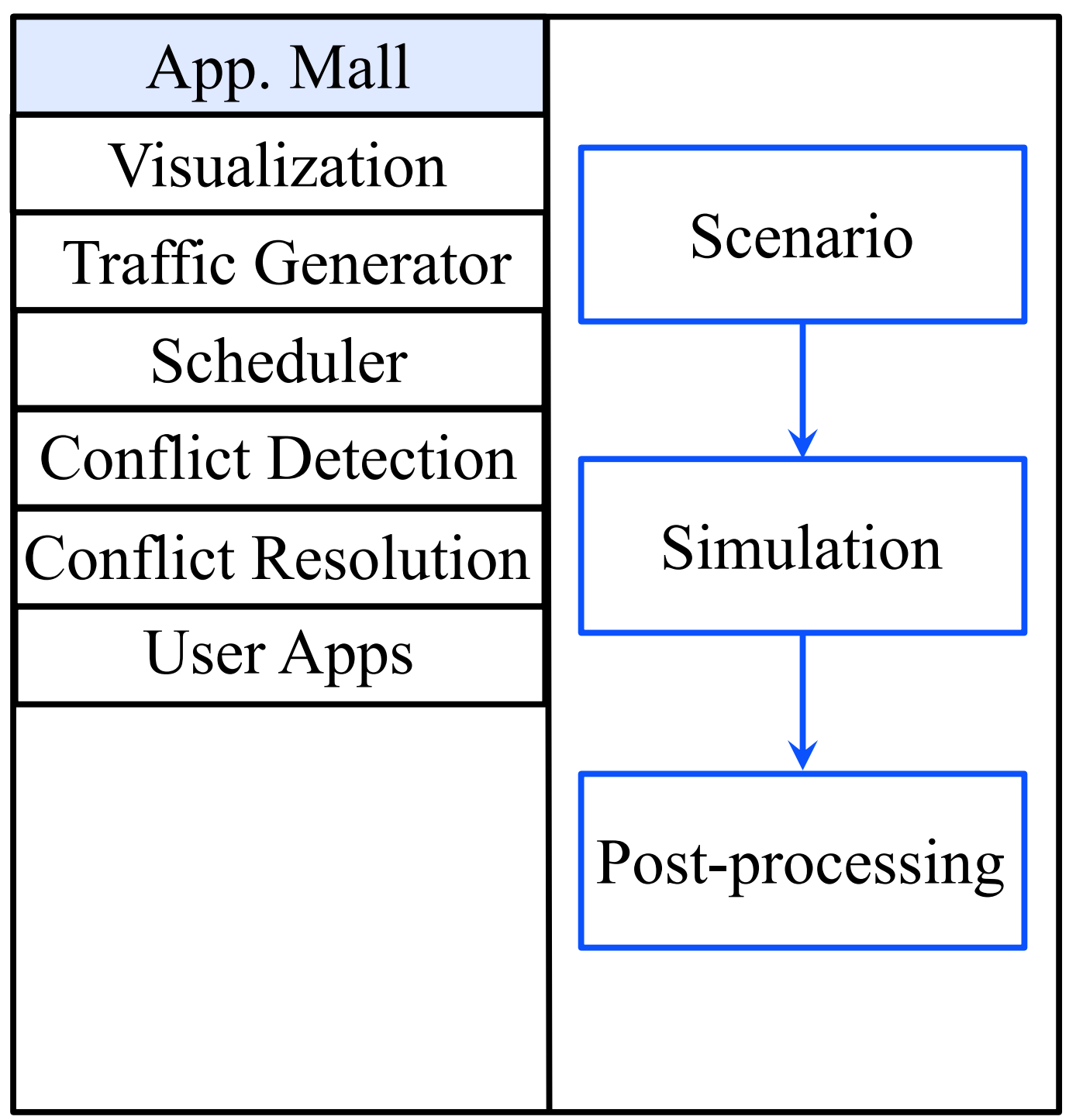

- NASA building testbed to accelerate deployment of ATM concepts into NAS

- Goal of providing access to community

- Provides infrastructure and some applications

- Scenario generation is one of the capabilities of testbed 


\section{Motivation}

- Manual creation of realistic scenarios for generating traffic for Human-in-the-Loop (HITL) simulation is difficult

- Missing and erroneous data

- Manual process is time consuming

- Difficulties cause studies to be limited to few scenarios

- Automated scenario generation has potential for overcoming limitations

- Use real air traffic data to create scenario

- Remove flights with erroneous data

- Select flights to achieve the desired short-haul to long-haul ratio

- Alter landing times to shape scenarios 


\section{Background: Aviation 2018 Paper}

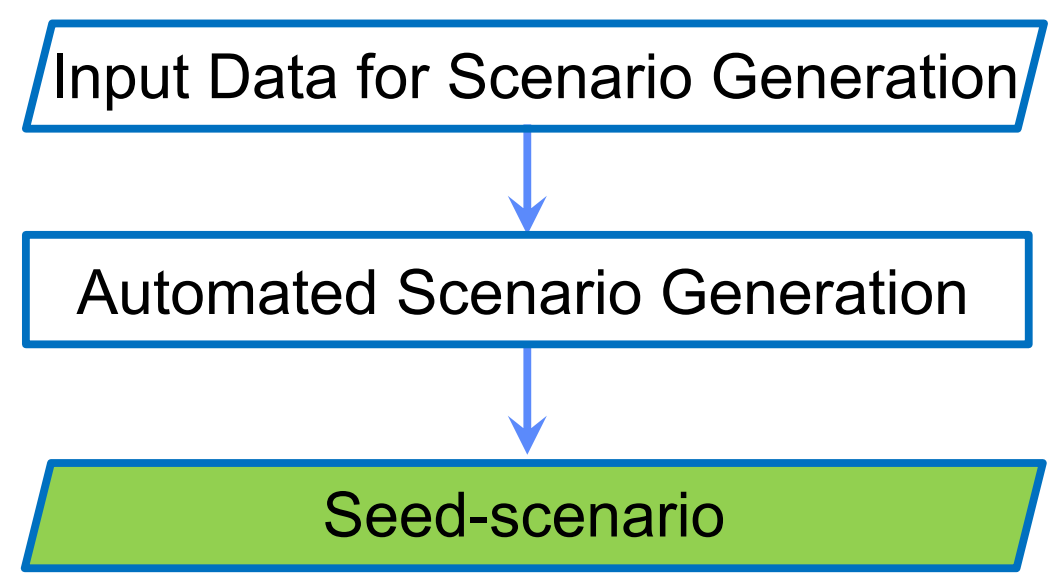

"Automated Scenario Generation for Human-in-the Loop Simulations," AIAA Modeling and Simulation Technologies Conference, Atlanta, GA June 25-29, 2018. 


\section{Background: Aviation 2018 Paper}

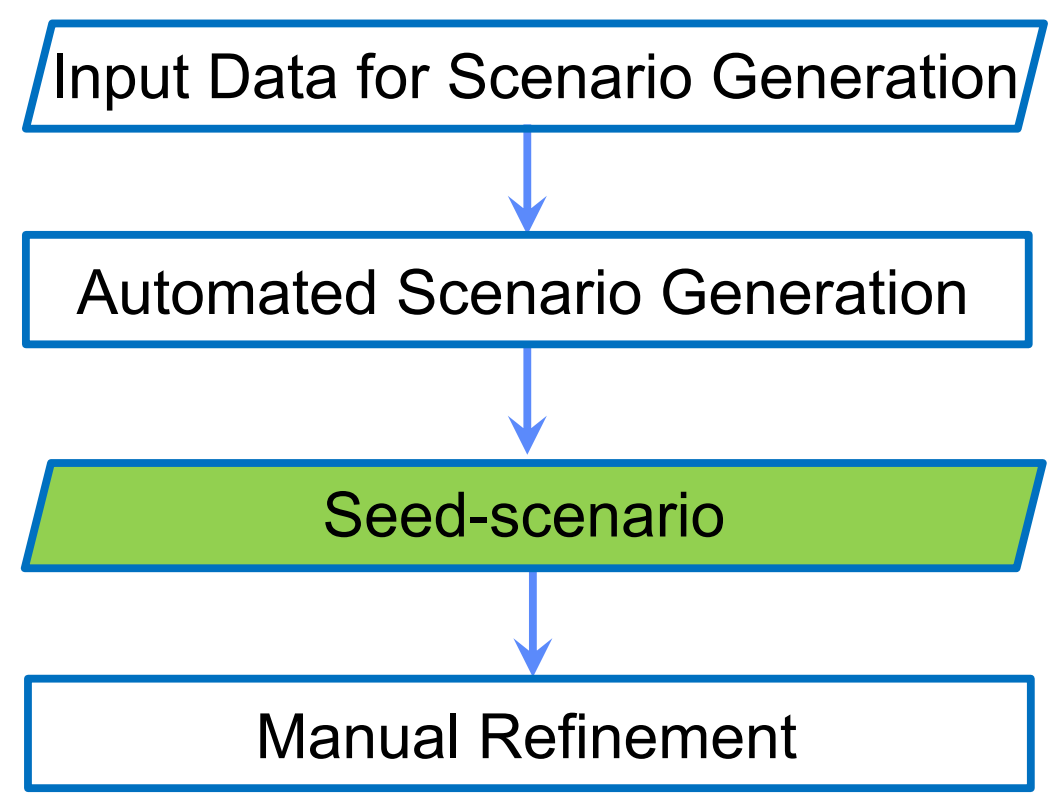




\section{Background: Aviation 2018 Paper}

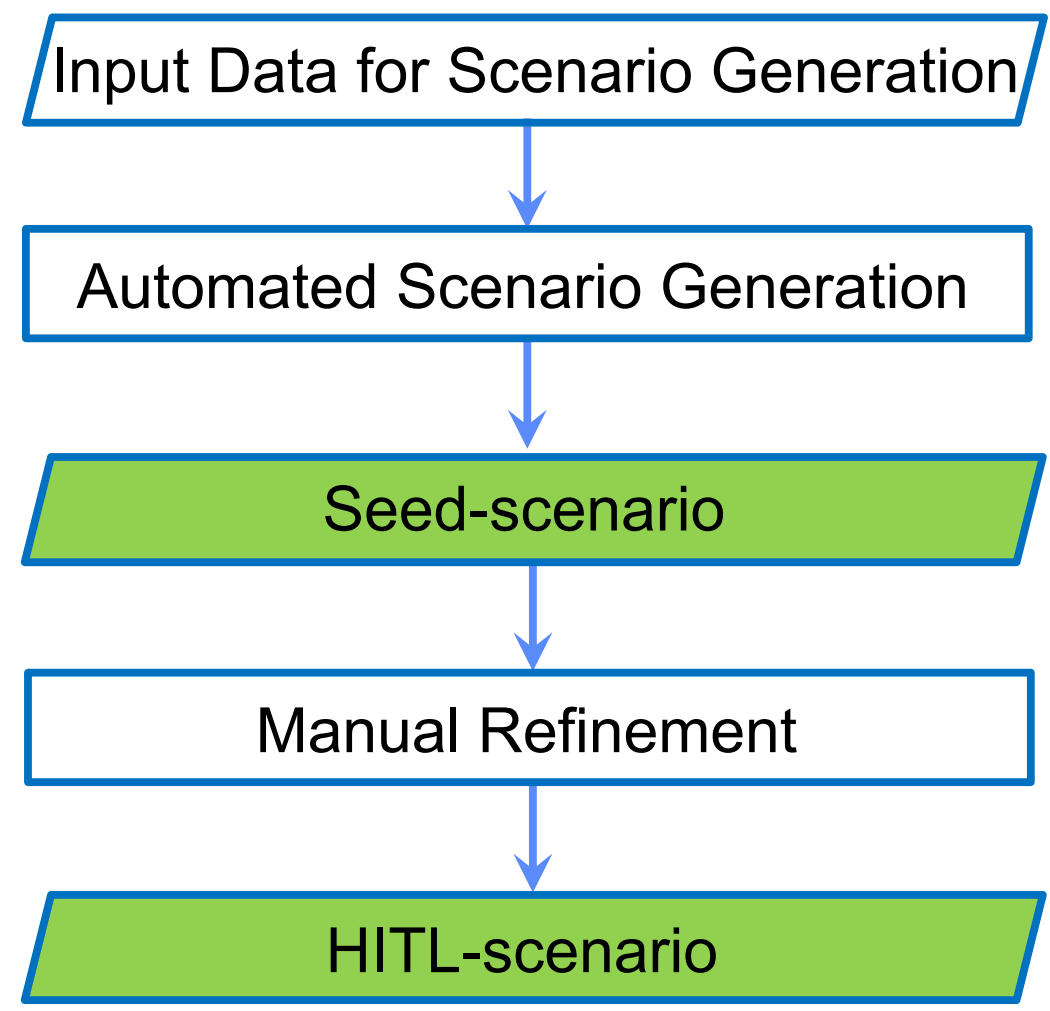

- $\quad$ Simulations can be run with ATM Testbed created seed-scenario

- $\quad$ Seed-scenario found to be a good starting point for creating HITL-scenario

- Experience showed that many of the manual adjustments can be automated to directly create the HITL-scenario 


\section{Outline}

- Step 1: Automated scenario generation using ATM Testbed

- Step 2: Automated scenario refinement

- Traffic scenario selection

- Results

- Conclusions 


\section{Input Data Source for Scenario Generation}

- System-Wide Information Management (SWIM) data processed into files, and stored in Sherlock data-warehouse

- Reduced Record (RD)

Single record for each flight

Beacon-code, flight-plan, takeoff/landing runway, departure/arrival time, sector/center transition list

- Event Data (EV)

Multiple records related to events for each flight

Event time and type- landing, crossing (sector, center, TRACON)

- Integrated Flight Format (IFF)

Multiple records for each flight

All flight plans including amended flight plans \& position data

- RD and EV useful for filtering and IFF for data augmentation 


\section{Multi-Aircraft Control System (MACS) Scenario Generation}

- MACS is a distributed system with multiple-pseudo pilot and air traffic controller stations

- It is frequently used at NASA for HITL evaluations of ATM concepts

- MACS traffic scenario consists of

- Flight route

- Initial conditions 
ATMTB Scenario Generation Steps

\author{
Load \& filter input data
}




\section{ATMTB Scenario Generation Steps}

Load \& filter input data

Preprocess flight data 


\section{ATMTB Scenario Generation Steps}

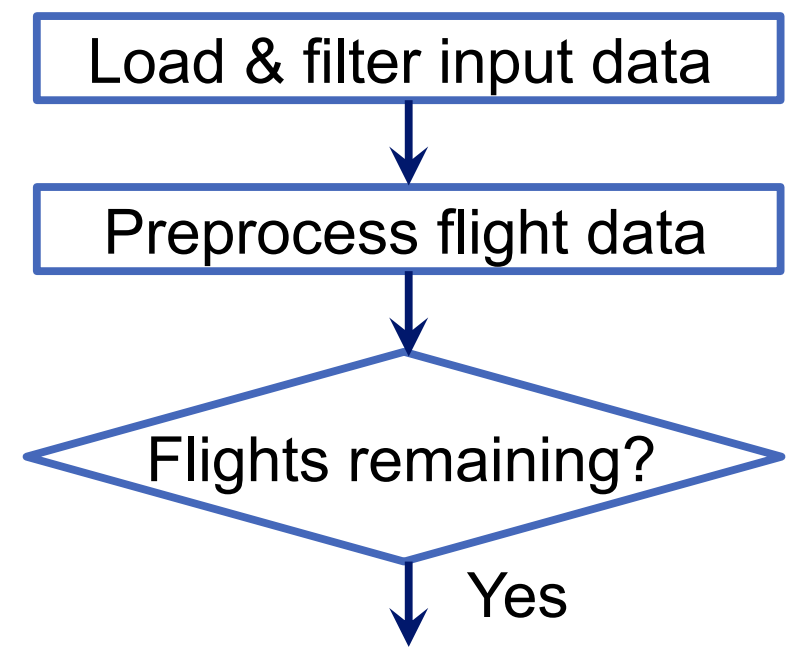




\section{ATMTB Scenario Generation Steps}

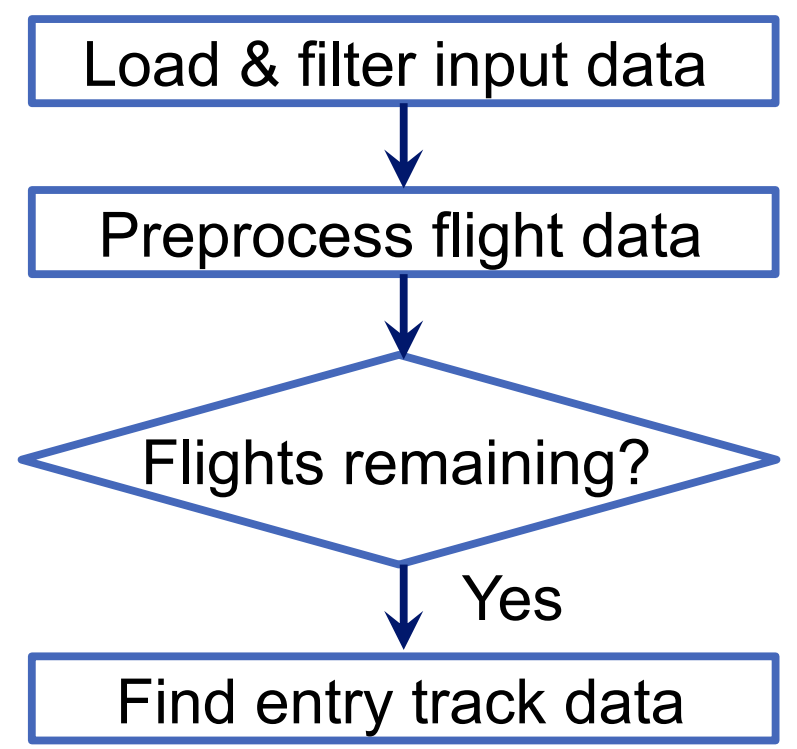




\section{ATMTB Scenario Generation Steps}

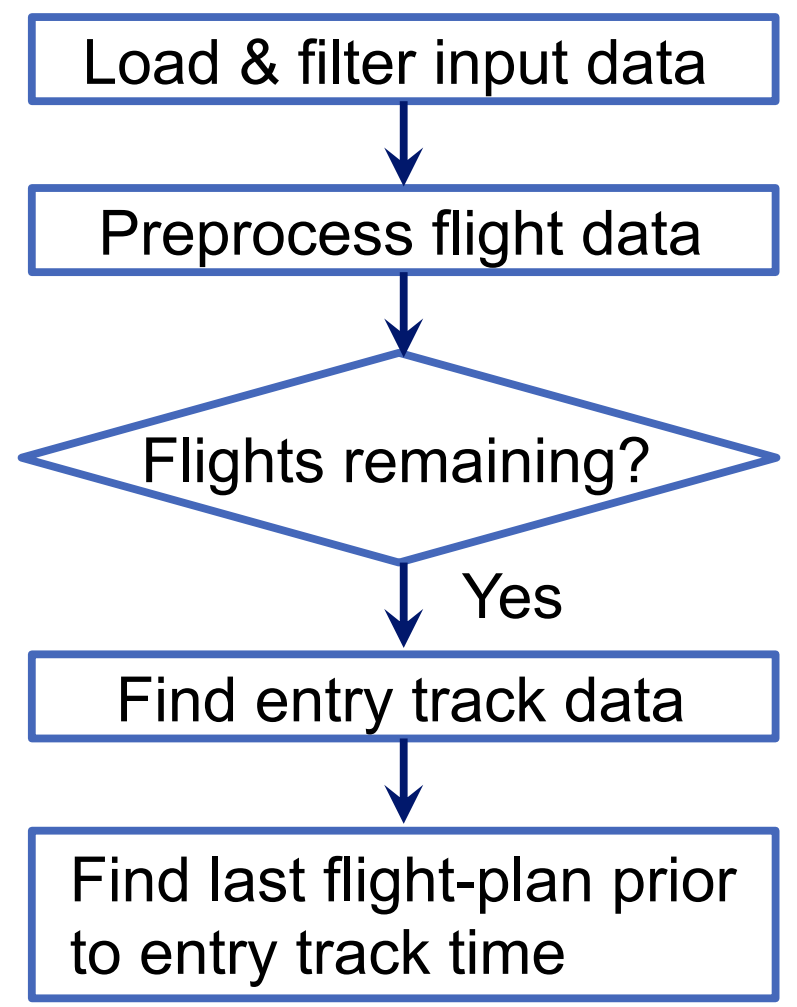




\section{ATMTB Scenario Generation Steps}

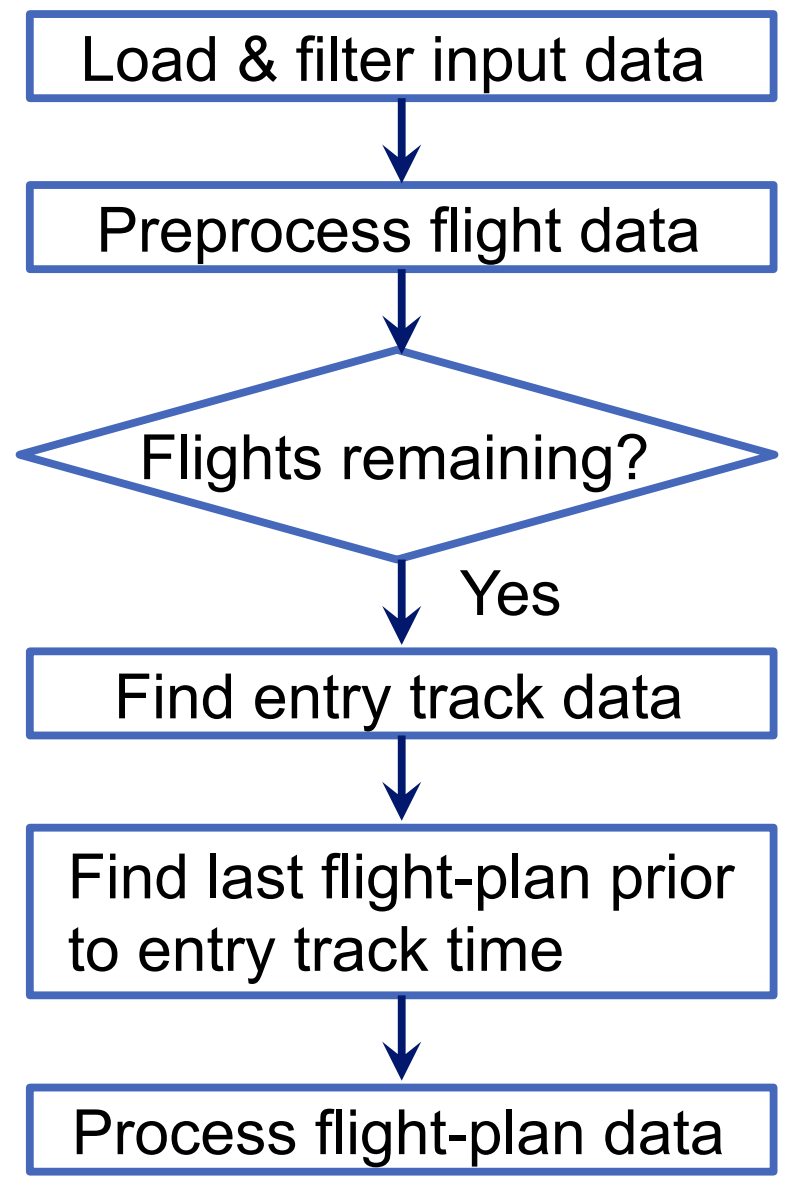




\section{ATMTB Scenario Generation Steps}

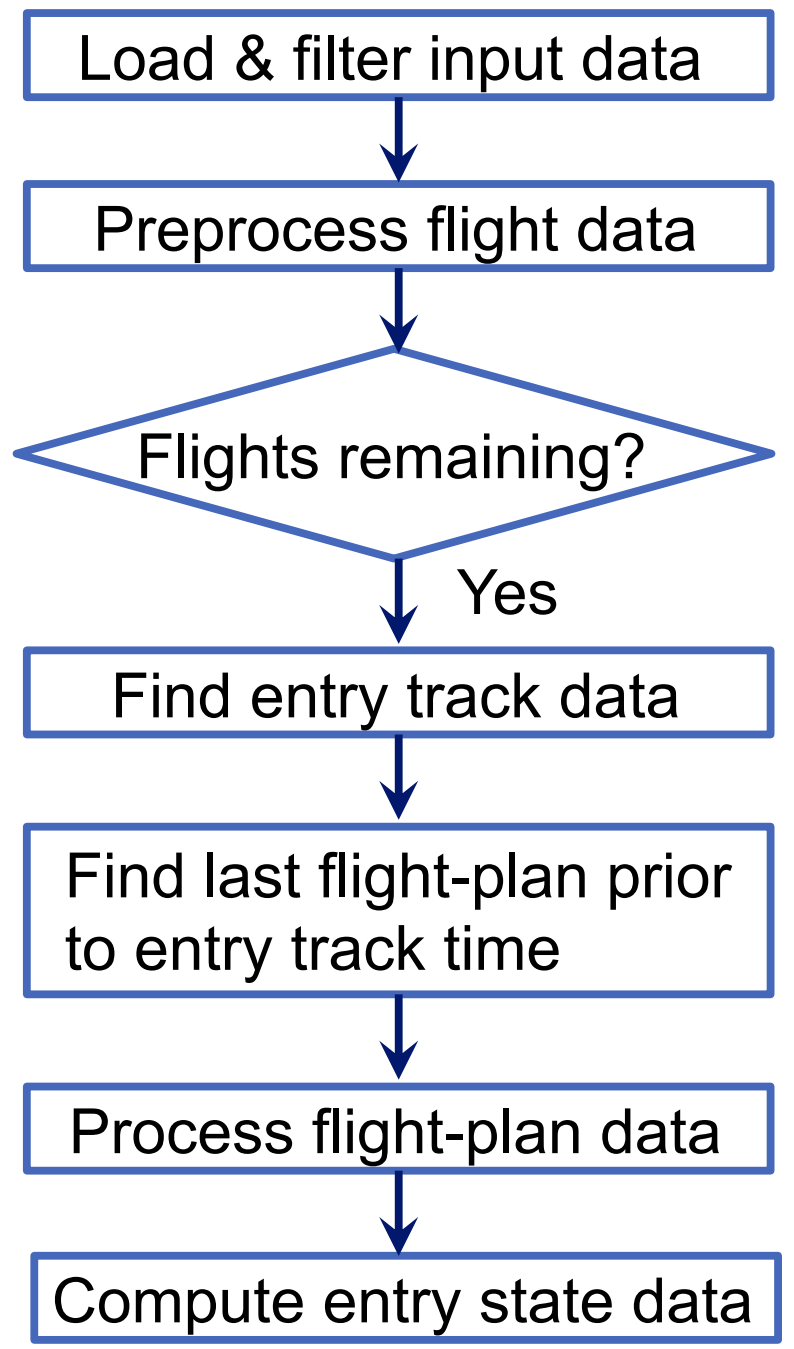




\section{ATMTB Scenario Generation Steps}

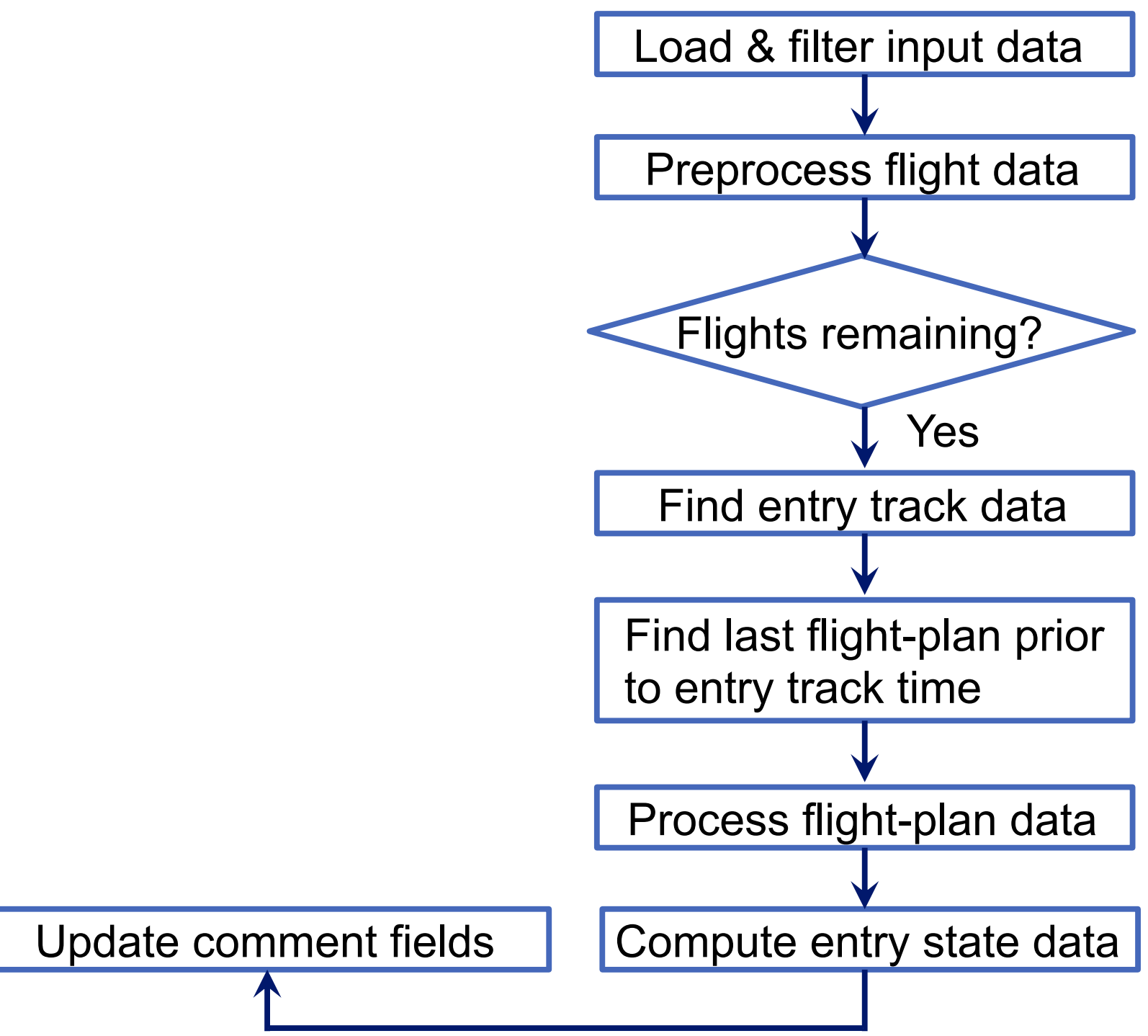




\section{ATMTB Scenario Generation Steps}

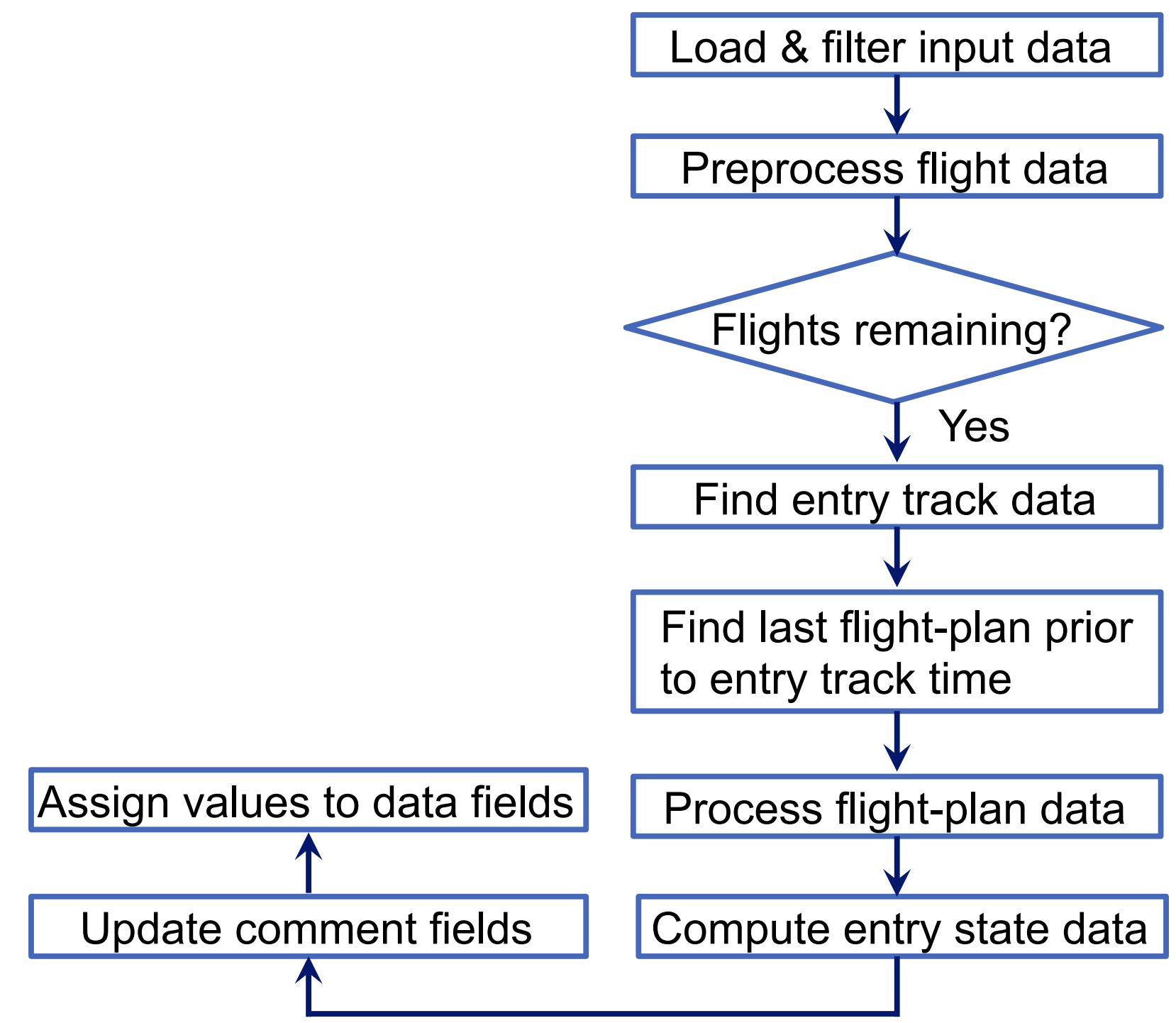




\section{ATMTB Scenario Generation Steps}

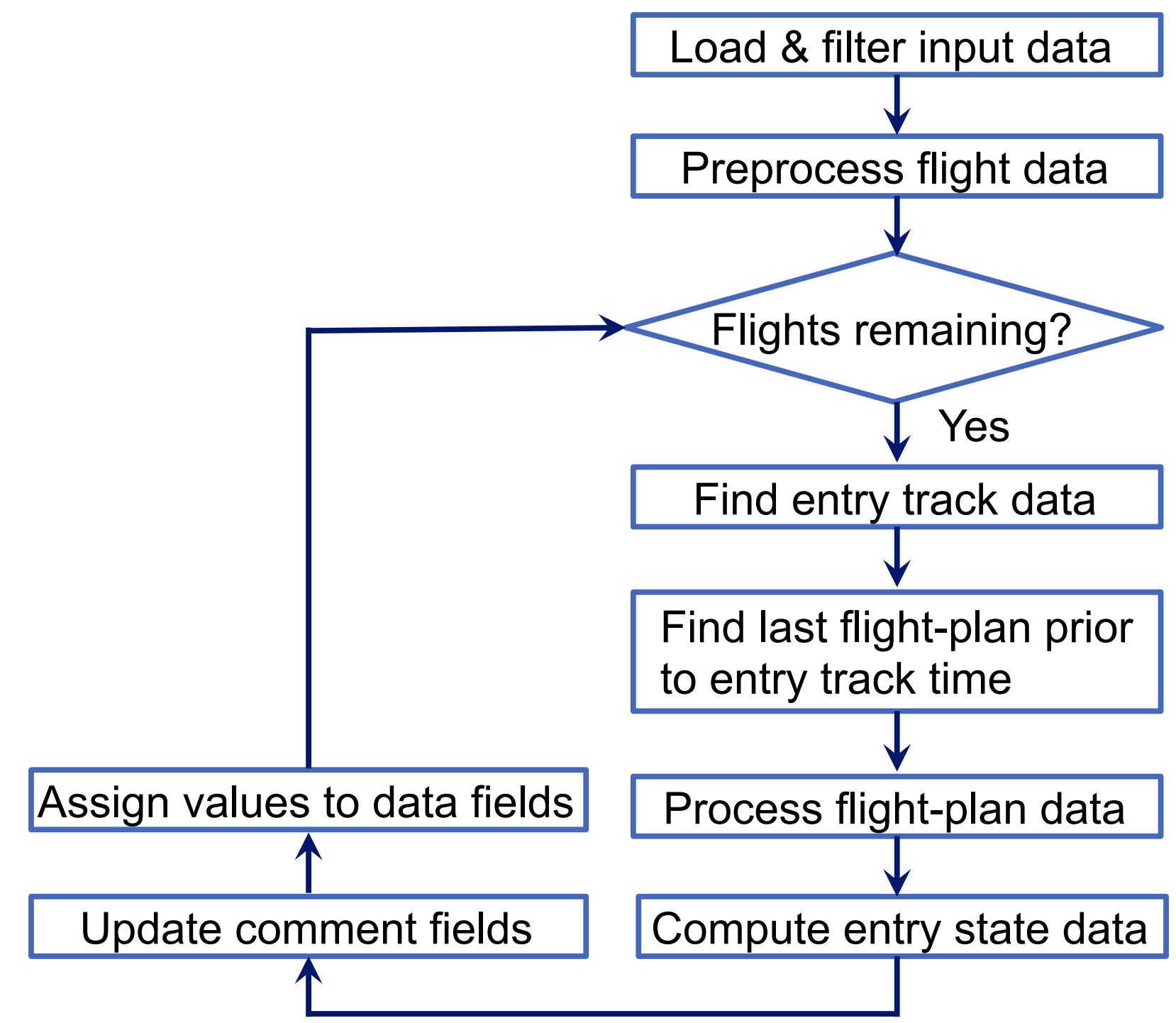




\section{ATMTB Scenario Generation Steps}

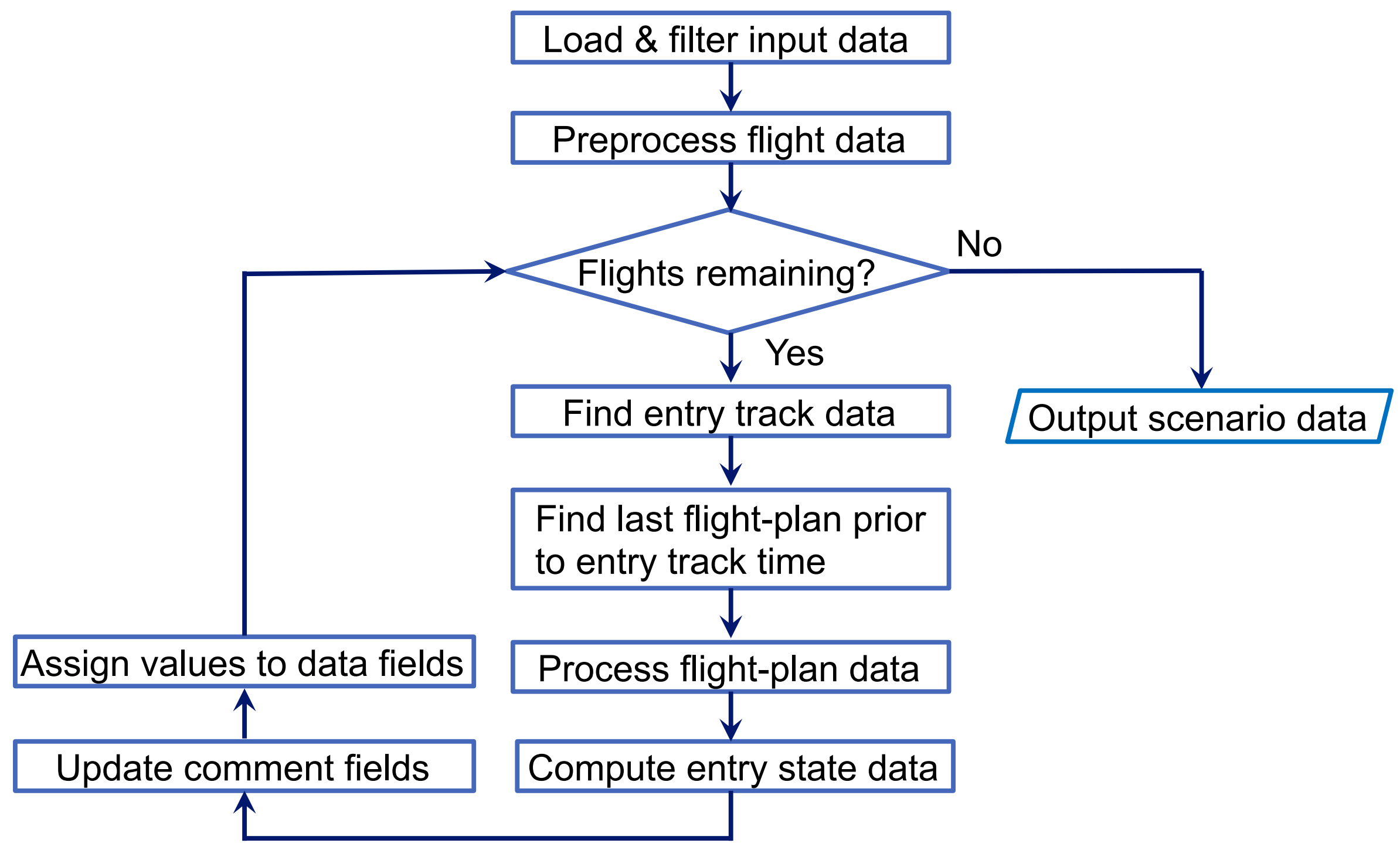




\section{ATMTB Scenario Generation Steps}

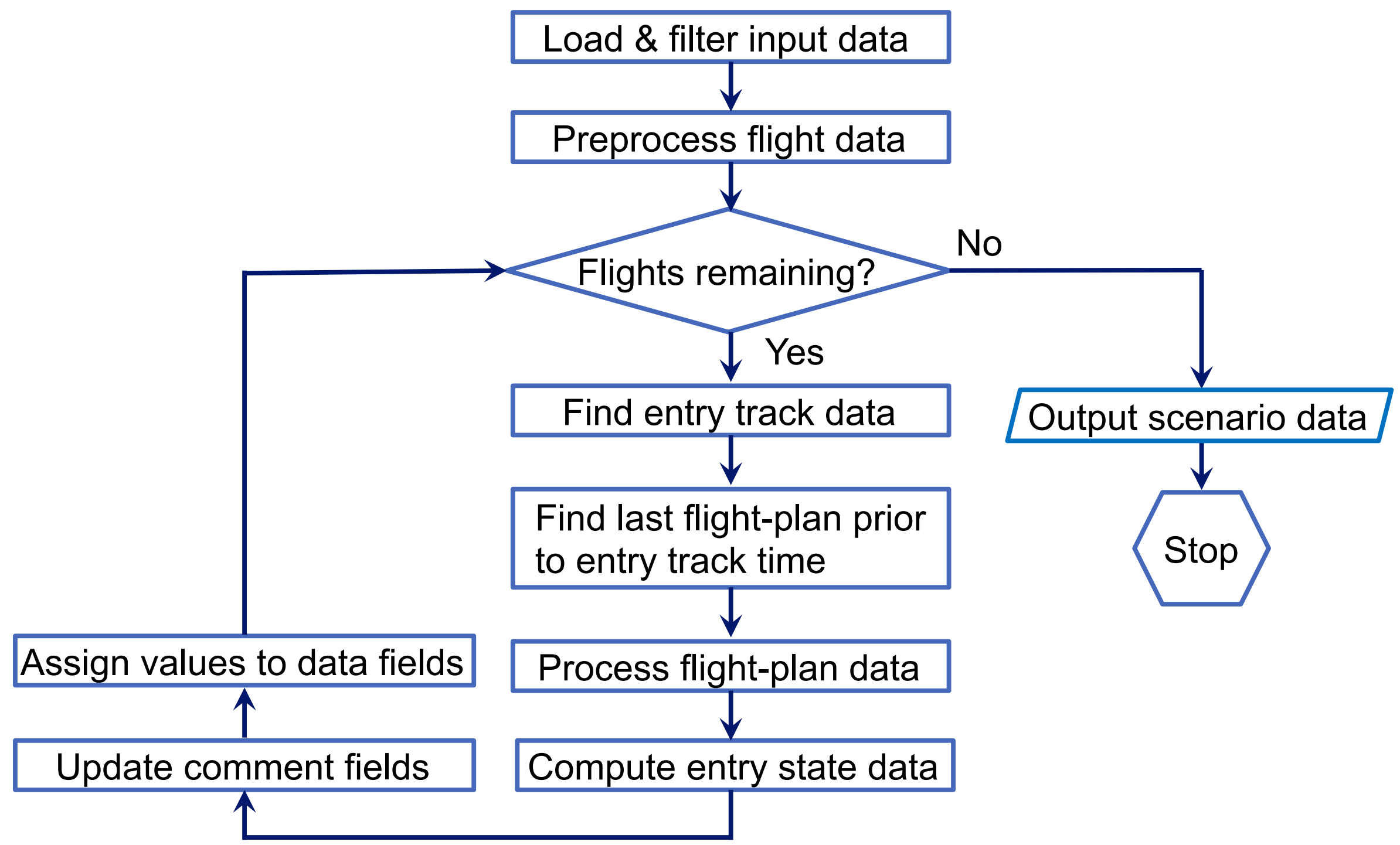


New Automated Scenario Generation Process

ATMTB generated scenario 


\section{New Automated Scenario Generation Process}

ATMTB generated scenario

1. Route length filter 


\section{New Automated Scenario Generation Process}

ATMTB generated scenario

1. Route length filter

2. Cruise speed filter 


\section{New Automated Scenario Generation Process}

ATMTB generated scenario

\begin{tabular}{|c|c|}
\hline 1. & Route length filter \\
\hline & \\
\hline 2. & Cruise speed filter \\
\hline & \\
\hline 3. & Cruise altitude filter \\
\hline
\end{tabular}




\section{New Automated Scenario Generation Process}

ATMTB generated scenario

\begin{tabular}{|c|c|}
\hline 1. & Route length filter \\
\hline & \\
\hline 2. & Cruise speed filter \\
\hline 3. & Cruise altitude filter \\
\hline & \\
\hline 4. & Entry time filter \\
\hline
\end{tabular}




\section{New Automated Scenario Generation Process}

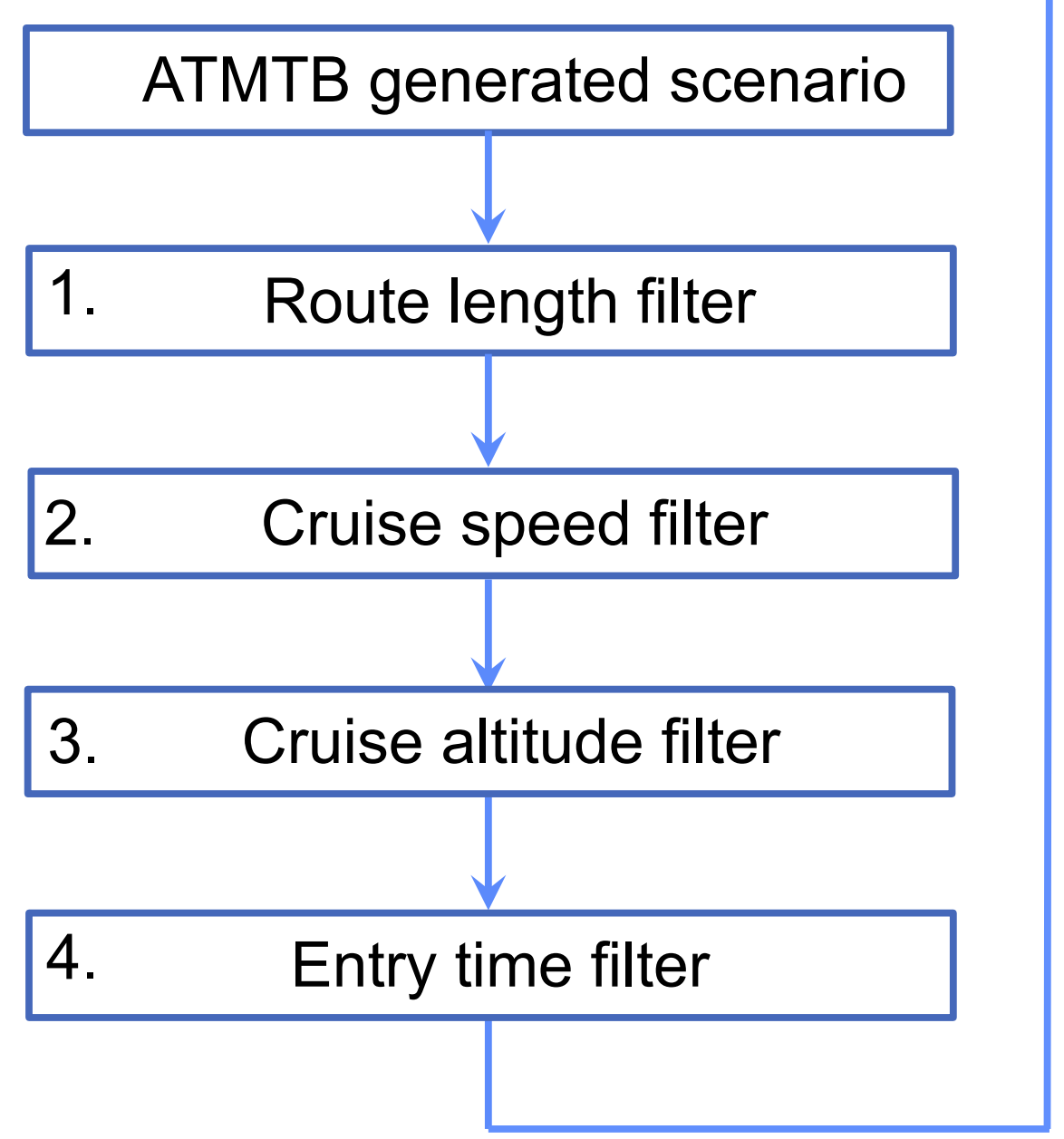




\section{New Automated Scenario Generation Process}
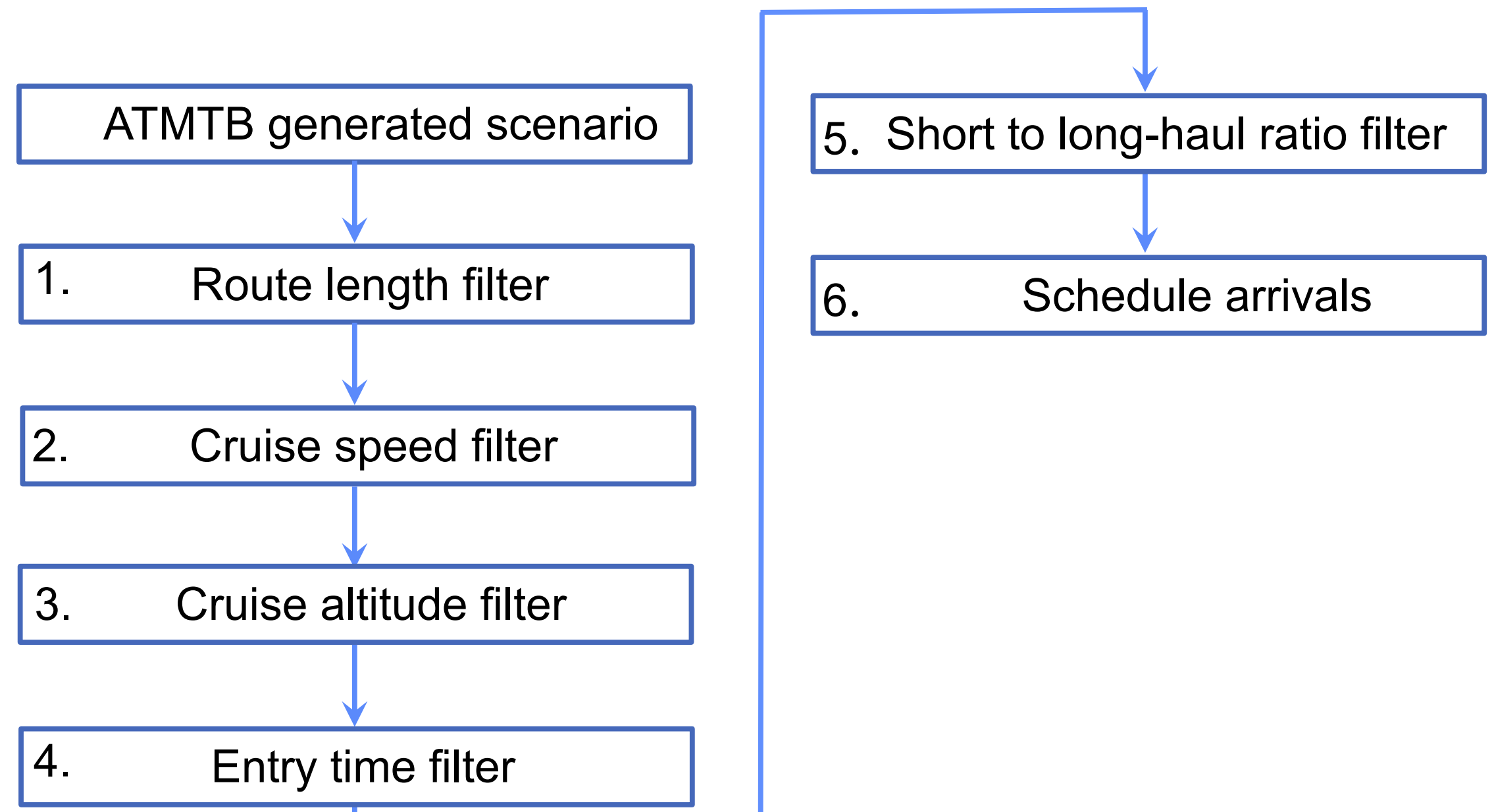


\section{New Automated Scenario Generation Process}
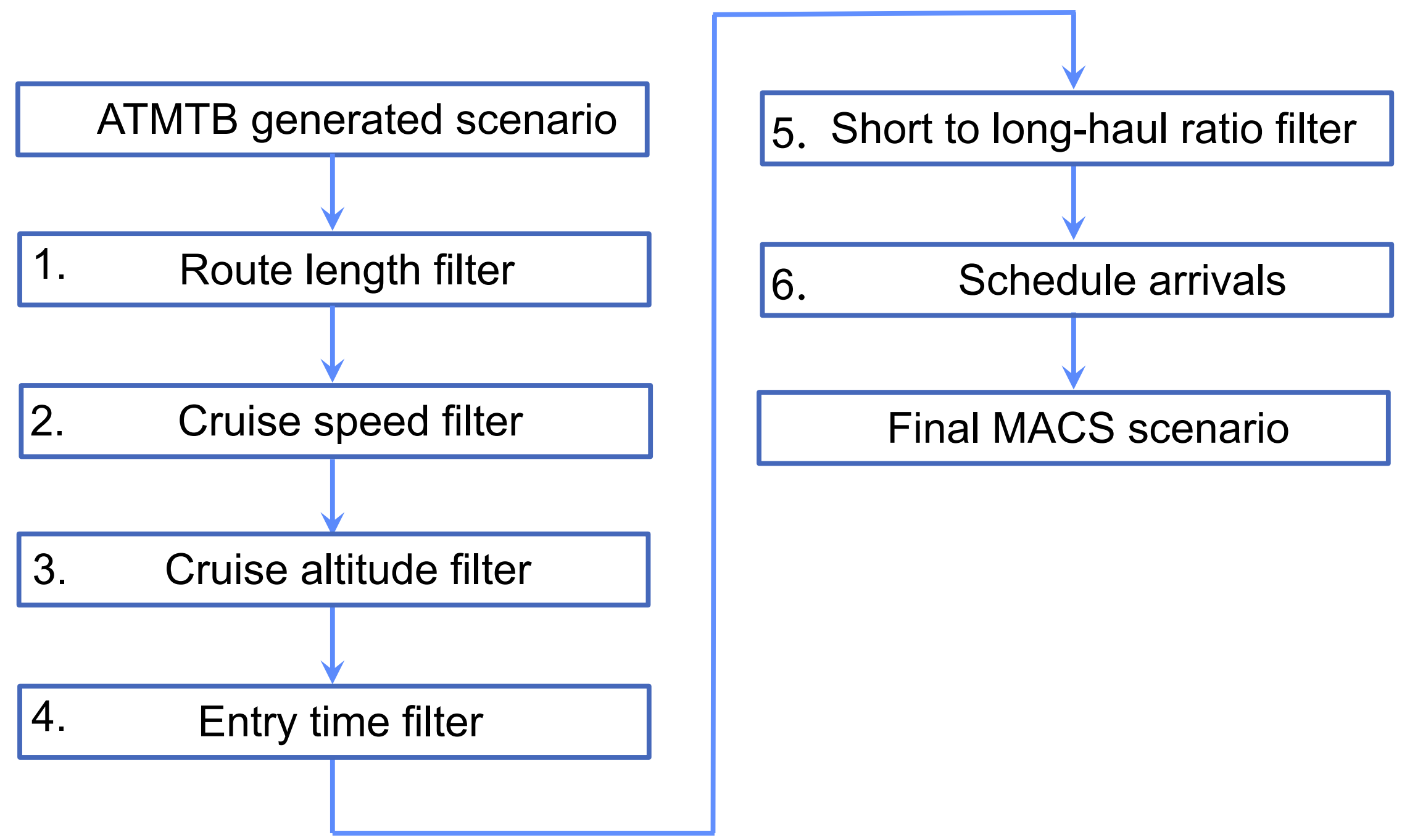


\section{Northeast Region Traffic Scenario}

- Obtained JFK, EWR, LGA and TEB runway configuration data from FAA's Aviation System Performance Metrics (ASPM) database

- Examined hourly JFK, EWR, LGA and TEB runway configuration data every day of 2017 to identify

- Most frequently used configurations individually

- Most frequently used configurations together

- Hours with the most operations taken together

- Selected 5/23/2017 for traffic scenario

- Chose six-hours from 18 UTC (14 local) to 23 UTC (19 local) 


\section{5/23/2017 Traffic Scenario}

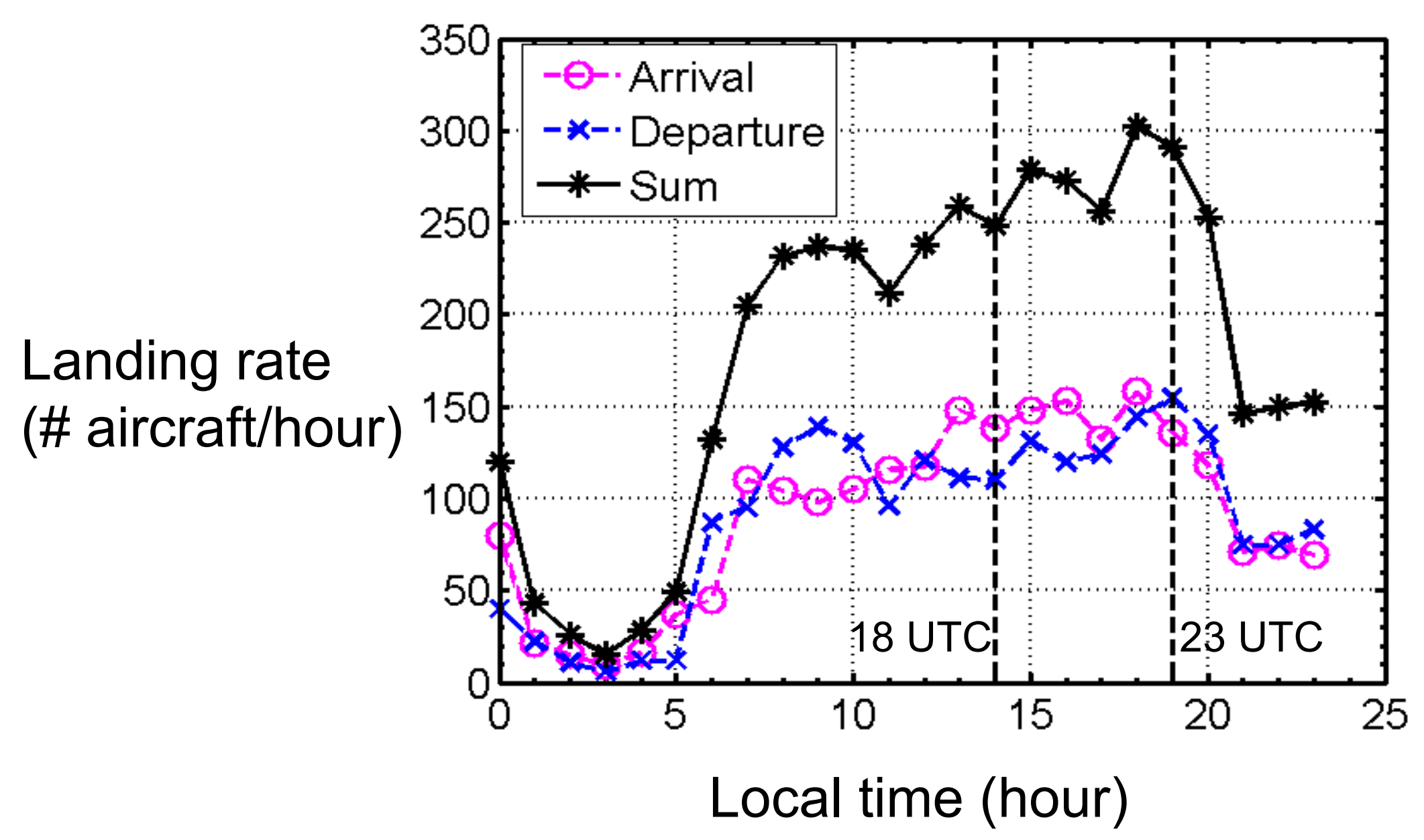




\section{Filtering Results}

\begin{tabular}{|r|r|r|}
\hline Filter & \multicolumn{1}{|c|}{ Criteria } & \# Aircraft \\
\hline ASPM & None & 865 \\
\hline ATMTB & MACS scenario generation & 808 \\
\hline Route length & $<20$ nautical-miles & 791 \\
\hline Cruise speed & $<120$ knots & 791 \\
\hline Cruise altitude & $<600$ feet & 769 \\
\hline Entry time & $<30$ minutes w.r.t start time & 769 \\
\hline
\end{tabular}




\section{Short-haul to Long-haul Ratio}

$x_{1}$ - \# long-hauls

$x_{2}$ - \# short-hauls

$x_{1 s}-\#$ selected long-hauls

$x_{2 s}-\#$ selected short-hauls

$r$-desired ratio

$$
\begin{aligned}
& \frac{x_{2 s}}{x_{1 s}}=r \\
& x_{1 s}=x_{1} \text { and } x_{2 s}=\left\lfloor r x_{1}\right\rfloor \text { if } r \leq \frac{x_{2}}{x_{1}} \\
& x_{1 s}=\left\lfloor\frac{x_{2}}{r}\right\rfloor \text { and } x_{2 s}=x_{2} \text { if } r>\frac{x_{2}}{x_{1}}
\end{aligned}
$$

\begin{tabular}{|r|r|r|r|}
\hline$\#$ & $r$ & Short-haul & Long-haul \\
\hline 1 & 0 & 0 & 531 \\
\hline 2 & 0.25 & 132 & 531 \\
\hline 3 & 0.5 & 238 & 476 \\
\hline 4 & 0.75 & 238 & 317 \\
\hline 5 & 1 & 238 & 238 \\
\hline 6 & 300 & 238 & 0 \\
\hline
\end{tabular}




\section{Scenario Landing Rate}

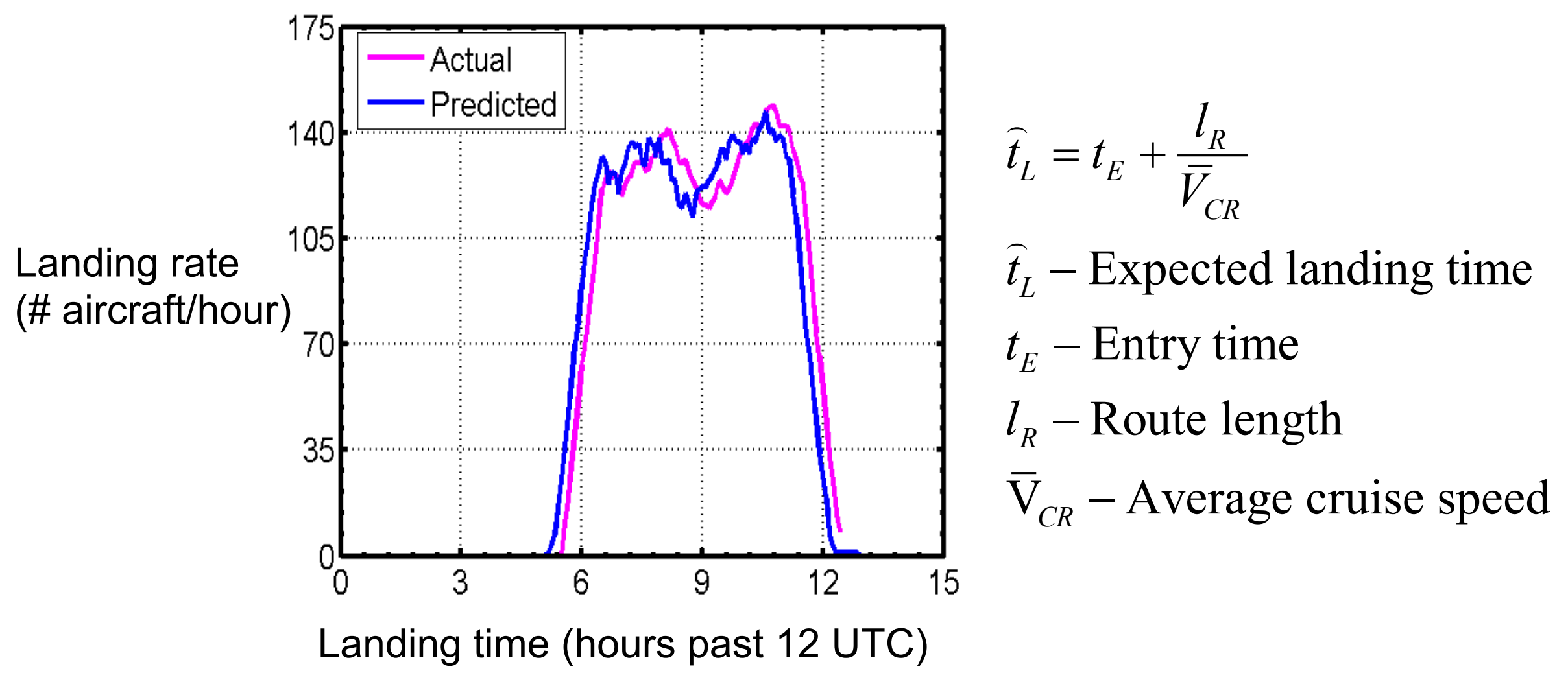




\section{Scheduling Arrivals}

$t_{p}-$ Proposed landing time

$t_{E}-$ Entry time

$t_{f}-$ Flight time

$t_{s}$-Scheduled landing time $t_{s E}$ - Scheduled entry time

$$
\begin{aligned}
& \Delta t=60 / \dot{n} \text { minutes } \\
& t_{s}(1)=t_{p}(1) \\
& t_{s}(i)=t_{s}(i-1)+\max \left(\Delta t, t_{p}(i)-t_{s}(i-1)\right) \\
& \delta(i)=\left(\Delta t-t_{p}(i)+t_{s}(i-1)\right)\left[t_{p}(i)-t_{s}(i-1)<\Delta t\right] \\
& t_{f}=t_{p}-t_{E} \\
& t_{s E}=t_{s}-t_{f} \\
& t_{s}(i)=t_{s}(i-1)+\Delta t
\end{aligned}
$$




\section{Results of Scheduling Arrivals}

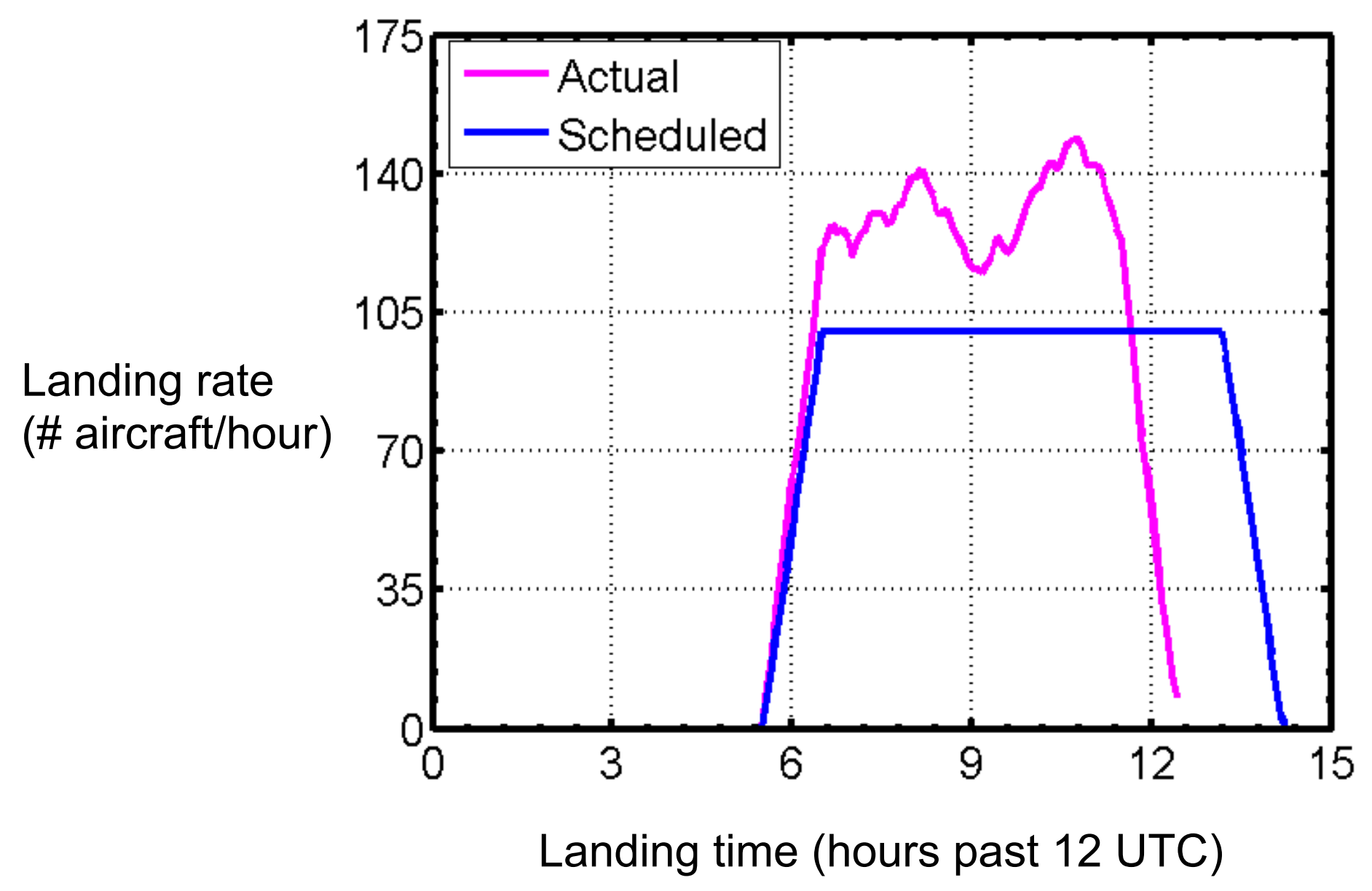




\section{Histograms of Entry Times}

Number of Flights

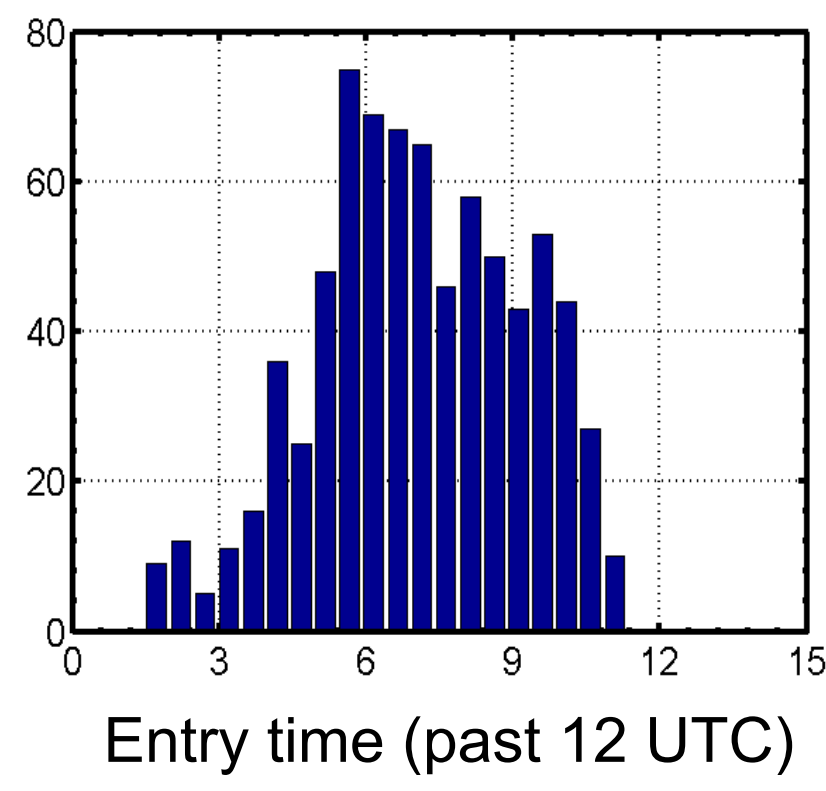

Original entry times

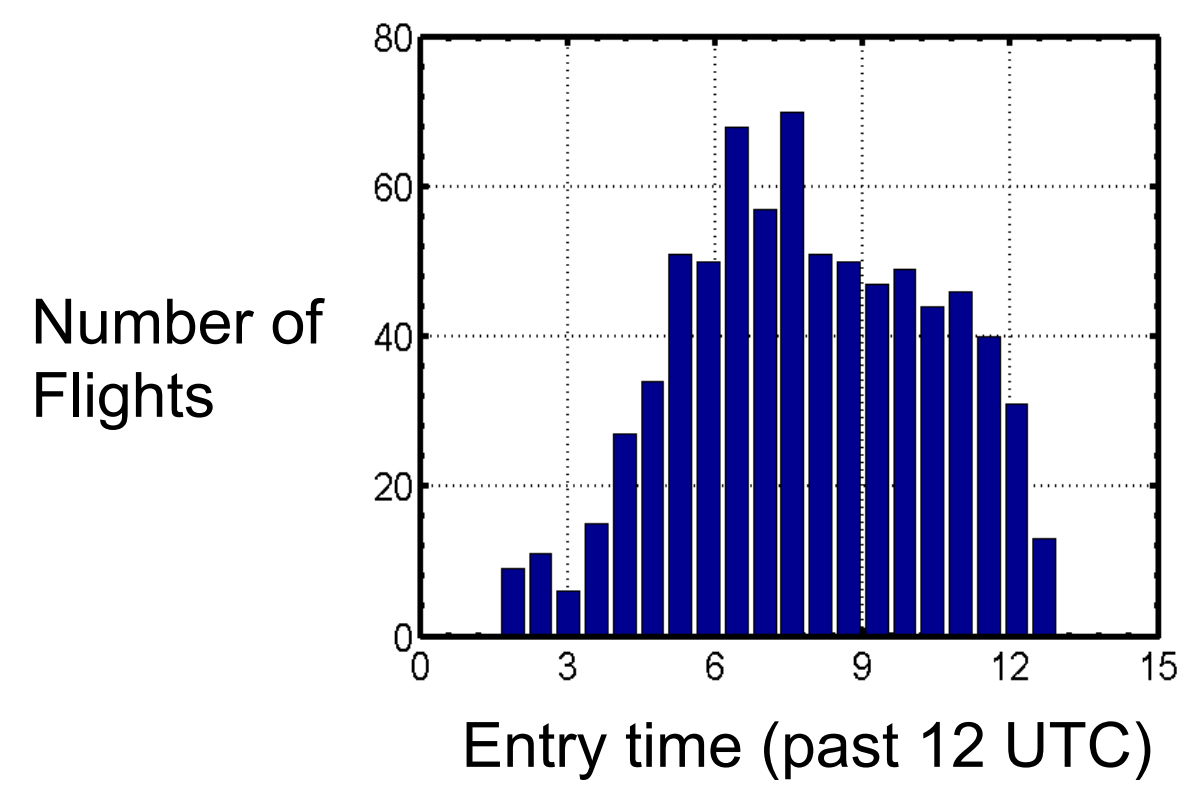

Scheduled entry times 


\section{Conclusions}

- New automated scenario generation process can create MACS scenarios for meeting HITL simulation requirements

- Selecting flights to achieve the desired short-haul to long-haul ratio

- Altering landing times

- The two-step process is

- Less error prone

- Faster and efficient

- Repeatable

- New process will be added to enhance ATMTB scenario generation capability 